Pacific Journal of Mathematics

THE WAVE EQUATION FOR DIFFERENTIAL FORMS 


\section{THE WAVE EQUATION FOR DIFFERENTIAL FORMS}

\section{AvNer FrIEdMaN}

1. The Problem. Let $M$ be a compact $C^{\infty}$ Riemannian manifold of dimension $N$, having a positive definite metric. The operator $\Delta=d \delta+$ $\delta d$ (see [13] for notation) maps $p$-forms $(0 \leqq p \leqq N)$ into $p$-forms and it reduces, when $p=0$, to minus the Laplace-Beltrami operator. Let $c(P)$ be a $C^{\infty}$ function which is nonpositive for $P \in M$, and consider the Cauchy problem of solving the system

$$
\begin{aligned}
& \left(L+\frac{\partial^{2}}{\partial t^{2}}\right) v \equiv\left(\Delta+c+\frac{\partial^{2}}{\partial t^{2}}\right) v=f(P, t) \\
& v(P, 0)=g(P), \quad \frac{\partial}{\partial t} v(P, 0)=h(P),
\end{aligned}
$$

where $f, g, h$ are $C^{\infty}$ forms of degree $p$. The main purpose of the present paper is to solve the system (1.1), (1.2) by the method of Fourier.

The Cauchy problem for second order self-adjoint hyperbolic equations was solved by Fourier's method by Ladyzhenskaya [8] and more recently (with some improvements) by V. A. Il'in [6]. In [8], other methods are also described, namely: finite differences, Laplace transforms, and analy tic approximations using a priori inequalities. Higher order hyperbolic equations were treated by Petrowski [12], Leray [9] and Garding [5].

The Fourier method can be based on the fact that the series

$$
\sum_{\lambda_{n}>0} \frac{\left|\varphi_{n}(x)\right|^{2}}{\lambda_{n}^{\alpha}}, \sum_{\lambda_{n}>0} \frac{\left|\partial \varphi_{n}(x) / \partial x\right|^{2}}{\lambda_{n}^{\alpha+1}}, \sum_{\lambda_{n}>0} \frac{\left|\partial^{2} \varphi_{n}(x) / \partial x^{2}\right|^{2}}{\lambda_{n}^{\alpha+2}}
$$

are uniformly convergent. Here $\left\{\varphi_{n}\right\}$ and $\left\{\lambda_{n}\right\}$ are the sequences of eigenfunctions and eigenvalues of the elliptic operator appearing in the hyperbolic equation. In [6] the convergence of (1.3) is proved for $\alpha=$ $[N / 2]+1$. Our proof of the analogous result for eigenforms is different from that of [6] and yields a better (and sharp) value for $\alpha$, namely, $\alpha=N / 2+\varepsilon$ for any $\varepsilon>0$. It is based on asymptotic formulas which we derive for $\sum_{\lambda \leq \lambda}\left|\partial^{j} \varphi_{n}(x) / \partial x^{j}\right|^{2}$ as $\lambda \rightarrow \infty$.

In $\S 2$ we recall various definitions and introduce the fundamental solution for $L+\partial / \partial t$ which was constructed by Gaffney [4] in the case $c(P) \equiv 0$. In $\S 3$ we derive some properties of the fundamental solution. These properties are used in $\S 4$ to derive the asymptotic formulas for $\sum_{\lambda_{n} \leqq \lambda}\left|\partial^{j} \varphi_{n}(x) / \partial x^{j}\right|^{2}$, by which the convergence of the series in (1.3) for any $\alpha>N / 2$ follows. In $\S 5$ we solve the problem (1.1), (1.2); first for $f, g, h$

Received January 13, 1961. Prepared under Contract Nonr 710 (16) (NR 044 004) between the Office of Naval Research and the University of Minnesota. 
infinitely differentiable and then under much weaker differentiability assumptions with regard to $M, c, f, g, h$. In $\S 6$ we briefly treat the Cauchy problem for the parabolic system

$$
\begin{gathered}
L u+\frac{\partial u}{\partial t}=f(P, t) \\
u(P, 0)=g(P) .
\end{gathered}
$$

2. Preliminaries. The first one to use fundamental solutions of the heat equation in the study of the asymptotic distributions of eigenvalues and eigenfunctions was Minakshisundaram [11]. Gaffney [4] extended his method to derive asymptotic formulas for eigenvalues and eigenforms. We shall describe here some well known facts and some of the results of [4] which we will need later on. Slight modifications will be made due to the fact that in [4] $c \equiv 0$.

As is well known, there exists a sequence of eigenvalues $\left\{\lambda_{n}\right\} \quad(0 \leqq$ $\lambda_{1} \leqq \cdots \leqq \lambda_{k} \rightarrow \infty$ as $\left.k \rightarrow \infty\right)$ and a sequence of the corresponding eigenforms $\left\{\omega_{n}\right\}$ of degree $p(0 \leqq p \leqq N, p$ is fixed throughout the paper) of $L$, that is, $L \omega_{n}=\lambda_{n} \omega_{n}$, such that the eigenforms form a complete orthonormal set in $L_{p}^{2}(M)$ (square integrable $p$-forms on $M$ ). The $\omega_{i}(p)$ are $C^{\infty}$ forms. The fundamental solution $\Theta(P, Q, t)$ of

$$
\left(L+\frac{\partial}{\partial t}\right) \omega=0
$$

is a double $p$-form which is twice differentiable in $Q$, once differentiable in $t$, satisfies (2.1) in $(Q, t), Q \in M, t>0$, (for any fixed $P$ ) and, for any $P \in M$,

$$
\lim _{t \rightarrow 0} \int_{M} \Theta(P, Q, t) * \alpha(Q)=\alpha(P)
$$

for any $L^{2} p$-form $\alpha$ which is continuous at $P$. As in [4] one easily derives the expansion (provided $\Theta$ is known to exist)

$$
\Theta(P, Q, t)=\sum_{i=1}^{\infty} \omega_{i}(P) \omega_{i}(Q) e^{-\lambda_{i} t}
$$

where the series on the right is pointwise convergent for all $P, Q \in M$, $t>0$ (that is, the series of each component is pointwise convergent).

A $p$-form $\alpha$ can be written locally as

$$
\alpha=\sum_{i_{1}<\ldots<i_{p}} A_{i_{1} \ldots i_{p}} d x^{i_{1}} \cdots d x^{i_{p}}=\Sigma^{\prime} A_{I} d x^{I}
$$

where' indicates summation on $I=\left(i_{1}, \cdots, i_{p}\right)$ with $i_{1}<\cdots<i_{p}$. The absolute value of $\alpha$ at $P$ is given by 


$$
|\alpha(P)|=\left[\Sigma^{\prime} A_{I}(x) A^{I}(x)\right]^{1 / 2}
$$

where $x$ is the local coordinate of $P$. Similarly, for a double $p$-form having local representation $\alpha(P, Q)=\Sigma^{\prime} A_{I J}(x, y) d x^{I} d y^{J}$ where $y$ is the local coordinate of $Q$, we define the absolute value by

$$
|\alpha(P, Q)|=\left[\sum_{I, J}^{\prime} A_{I J}(x, y) A^{I J}(x, y)\right]^{1 / 2} .
$$

The right "half-norm" is defined by

$$
|\alpha| \mid(P)=\left[\int_{M}|\alpha(P, Q)|^{2} d V_{Q}\right]^{1 / 2} .
$$
by

Given two double $p$-forms $\alpha$ and $\beta$, a new double $p$-form is defined

$$
[\alpha, \beta]=[\alpha, \beta](P, Q)=\int_{M} \alpha(P, W) * \beta(Q, \mathrm{~W}) .
$$

One then verifies:

$$
|[\alpha, \beta](P, Q)| \leqq|\alpha\|(P) \mid \beta\|(Q) .
$$

The following inequalities are immediate:

$$
|\alpha+\beta| \leqq|\alpha|+|\beta|,|\alpha+\beta||\leqq| \alpha||+|\beta| \mid,
$$

where $\alpha, \beta$ are any double $p$-forms.

In order to construct $\Theta$, one first constructs a parametrix. Gaffney [4] constructs a parametrix by generalizing the method of Minakshisandaram [11], making use of some calculation of Kodaira [7]. Given a point $P$, let $y=\left(y^{i}\right)$ be normal coordinates about $P$ (with coordinates $\left.x^{i}\right)$. A $p$-form can be written as a vector $X$ with $\left(\begin{array}{l}N \\ p\end{array}\right)$ components and then

$$
\Delta X=-\Sigma g^{i j} \partial_{i} \partial_{j} X+\Sigma A^{i} \partial_{i} X+B X
$$

where $\left(g_{i j}\right)$ is the metric tensor, $\left(g^{i j}\right)$ is the inverse matrix, $\partial_{i}=\partial / \partial x^{i}$, and $A^{i}, B$ are matrices depending on the $g_{i}$ and their first two derivatives. If $X=f\left(r^{2}\right) W(x, y)$ where $r$ is the geodesic distance from $x$ to $y$ (each component of $X$ is now a vector so that $W$ is a square matrix), then

(2.7) $\Delta_{y}\left[f\left(r^{2}\right) W\right]=f\left(r^{2}\right) \Delta_{y} W-f^{\prime}\left(r^{2}\right)\left\{2 N-4 K+4 r \frac{\partial}{\partial r}\right\} W-4 r^{2} f^{\prime \prime}\left(r^{2}\right) W$, where $K=K(x, y)$ is a $C^{\infty}$ matrix which vanishes for $y=x$.

There exists a $C^{\infty}$ matrix $M$ satisfying

$$
r \frac{\partial}{\partial r} M=K M(x \text { fixed }), \quad M(x, x)=I
$$


where $I$ is the identity matrix. Using (2.8), (2.7) is simplified to

$$
M^{-1} \Delta_{y}(f M W)=f\left(M^{-1} \Delta M\right)_{y} W-f^{\prime}\left\{2 N+4 r \frac{\partial}{\partial r}\right\} W-4 r^{2} f^{\prime \prime} W .
$$

(2.9) will now be applied with

$$
f\left(r^{2}, t\right)=\frac{1}{(4 \pi t)^{N / 2}} e^{-r^{2} / 4 t} \quad(t>0 \text { fixed }) .
$$

Setting

$$
H_{m}=\sum_{j=0}^{m} f M U_{j} t^{j}, \quad U_{0}=I
$$

one then gets

$$
\Delta H_{\infty}=f M \sum_{j=0}^{\infty}\left\{\left(M^{-1} \Delta M\right) U_{j} t^{j}+\frac{1}{4 t}\left(2 N+4 r \frac{\partial}{\partial r}\right) U_{j} t^{\jmath}-\frac{r^{2}}{4 t^{2}} U_{j} t^{\jmath}\right\} .
$$

Calculating also $\partial H_{\infty} / \partial t$, one then obtains

$$
\left(L_{y}+\frac{\partial}{\partial t}\right) H_{\infty}=f M \sum_{j=0}^{\infty}\left\{\left(M^{-1} \Delta M+c\right) U_{j}+\left(r \frac{\partial}{\partial r}+j+1\right) U_{j+1}\right\} t^{j}
$$

which leads to the successive definitions:

$$
U_{j}=-\frac{1}{r^{j}} \int_{0}^{r}\left(M^{-1} \Delta M+c\right) U_{j-1} d r(1 \leqq j<\infty), \quad \text { where } \quad U_{0}=I .
$$

We conclude that, for any $m \geqq 0$,

$$
\left(L_{y}+\frac{\partial}{\partial t}\right) H_{m}=\frac{1}{(4 \pi)^{N / 2}} e^{-r^{2} / 4 t} t^{m-N / 2} L_{y}\left(M U_{m}\right) .
$$

$H_{m}$ is a local parametrix. Note that when $P, Q$ vary in a sufficiently small neighborhood $V$ (contained in one coordinate patch), $H_{m}$ is defined and is $C^{\infty}$ in $(P, Q, t)$ if $t>0$. Let $\eta_{\varepsilon}(r)$ be a $C^{\infty}$ function of $r$ which is equal to 1 for $r<\varepsilon$ and is equal to 0 for $r>2 \varepsilon$. If $\varepsilon$ is sufficiently small then the support of $\eta_{\varepsilon}(r) H_{m}(P, Q, t)$ (where $r$ is the distance from $P$ to $Q$ ) as a form in $Q$ lies in $V$, provided $P \in W$, where $W$ is a given open subset of $V, \bar{W} \subset V$. We can cover the manifold $M$ by a finite number of sets $W$, call then $W_{i}$. Let the $H_{m}$ corresponding to (the corresponding) $V_{i}$ be denoted by $H_{m}^{i}$. If $\left\{\alpha_{i}\right\}$ is a $C^{\infty}$ partition of unity subordinate to $\left\{W_{i}\right\}$, then the support of $\alpha_{i}(P) \eta_{\varepsilon}(r) H_{m}^{i}(P, Q, t)$ as a form of $(P, Q)$ lies in $W_{i} \times V_{i}$ and hence this form is $C^{\infty}$ in $(P, Q, t)$ if $t>0$. The global parametrix is given by

$$
\Theta_{m}(P, Q, t)=\Sigma \alpha_{i}(P) \eta_{\mathrm{\varepsilon}}(r) H_{m}^{i}(P, Q, t) .
$$


The fundamental solution should then formally be

$$
\Theta(P, Q, t)=\Theta_{m}(P, Q, t)+\int_{0}^{t}\left[\gamma_{m}(P, U, t), \Theta_{m}(Q, U, t-\tau)\right] d \tau
$$

where $\gamma_{m}$ is defined by

$$
\begin{gathered}
\gamma_{m}(P, Q, t)=\sum_{i=1}^{\infty}(-1)^{i} \delta_{m}^{i}(P, Q, t) \\
\delta_{m}^{i}(P, Q, t)=\int_{0}^{t}\left[\delta_{m}^{i-1}(P, U, \tau), \delta_{m}^{i}(Q, U, t-\tau)\right] d \tau, \\
\delta_{m}^{1}=\left(L_{y}+\frac{\partial}{\partial t}\right) \Theta_{m} .
\end{gathered}
$$

Using (2.4) and the inequality

$$
\left|\int_{0}^{t} \alpha(P, Q, \tau) d \tau\right| \leqq\left(\begin{array}{c}
N \\
p
\end{array}\right) \int_{0}^{t}|\alpha| d \tau,
$$

Gaffney establishes the uniform convergence of the right side of (2.14) and then proves that $\Theta$, as defined in (2.13), is a fundamental solution, for any $m \geqq 0$, written in matrix form. We shall use the matrix notation of $\Theta$ and the usual double form notation for $\Theta$ interchangably; the same for $\Theta_{m}$.

3. Properties of the fundamental solution. We denote by $\partial_{P}^{h} \Theta(P, Q, t)$ an $h$ th derivative of $\Theta$ with respect to the coordinates of $P$, in a given coordinate system. If $h=\left(h_{1}, \cdots, h_{N}\right)$, set $|h|=h_{1}+\cdots+h_{N}$. From the formulas defining $\Theta$ it is clear that $\partial_{P}^{h} \Theta(P, Q, t)$ exists and is continuous (in fact $C^{\infty}$ ) in $P, Q \in M$ and $t>0$. Let

$$
\partial_{P}^{h} \Theta(P, Q, t) \sim \sum_{i=1}^{\infty} B_{i}(P, t) \omega_{i}(Q)
$$

be the Fourier expansion of $\partial_{p}^{h} \Theta$, for $(P, t)$ fixed. Then (recalling (2.3))

$$
\begin{aligned}
B_{i}(P, t) & =\int_{M} \partial_{P}^{h} \Theta(P, U, t) * \omega_{i}(U)=\partial_{P}^{h} \int_{M} \Theta(P, U, t) * \omega_{i}(U) \\
& =\partial^{h} \omega_{i}(P) e^{-\lambda_{i} t},
\end{aligned}
$$

where $\partial_{P}^{h}$ is abbreviated by $\partial^{h}$ when there is no confusion.

By the (easily verified) Parseval's equality we get

$$
\begin{aligned}
\psi(P, Q, t) & \equiv\left[\partial_{P}^{h} \Theta\left(P, U, \frac{t}{2}\right), \partial_{Q}^{h} \Theta\left(Q, U, \frac{t}{2}\right)\right] \\
& =\sum_{i=1}^{\infty} \partial_{P}^{h} \omega_{i}(P) \partial_{Q}^{h} \omega_{i}(Q) e^{-\lambda_{i} t}
\end{aligned}
$$

and the series is pointwise convergent for $P, Q \in M, t>0$. 
We need the following notations. Let $\alpha$ be a double $p$-form. If it is locally represented by $\Sigma^{\prime} A_{I J} d x^{I} d y^{J}$, then we set

$$
[\alpha(P, P)]=\Sigma^{\prime} A_{I}^{I} \text {. }
$$

If $\beta$ is also a double $p$-form, then we define $\left[[\alpha(P, U), \beta(P, U)]_{U}\right]$ to be $[\gamma(P, P)]$ where $\gamma(P, Q)=[\alpha(P, U), \beta(Q, U)]$.

Using (2.13) and the definition of $\psi$ in (3.3) we have

$$
\begin{aligned}
\sum_{i=1}^{\infty} \mid & \left.\partial^{h} \omega_{i}(P)\right|^{2} e^{-\lambda_{i} t}=[\psi(P, P, t)] \\
= & {\left[\left[\partial_{P}^{h} \Theta_{m}\left(P, U, \frac{t}{2}\right), \partial_{P}^{h} \Theta_{m}\left(P, U, \frac{t}{2}\right)\right]_{U}\right] } \\
& +2\left[\left[\int_{0}^{t / 2}\left[\partial_{P}^{h} \gamma_{m}(P, W, \tau), \Theta_{m}\left(U, W, \frac{t}{2}-\tau\right)\right] d \tau, \partial_{P}^{h} \Theta_{m}\left(P, U, \frac{t}{2}\right)\right]_{U}\right] \\
& +\left[\left[\int_{0}^{t / 2}\left[\partial_{P}^{h} \gamma_{m}(P, W, \tau), \Theta_{m}\left(U, W, \frac{t}{2}-\tau\right)\right] d \tau,\right.\right. \\
& \left.\left.\int_{0}^{t / 2}\left[\partial_{P}^{h} \gamma_{m}(P, W, \tau), \Theta_{m}\left(U, W, \frac{t}{2}-\tau\right)\right] d \tau\right]_{U}\right] \\
\equiv & J_{1}(P, t)+2 J_{2}(P, t)+J_{3}(P, t) .
\end{aligned}
$$

We proceed to estimate the $J_{i}$. We shall make use of the inequality [4]

$$
[\alpha(P, P)] \leqq\left(\begin{array}{c}
N \\
p
\end{array}\right)|\alpha(P, P)|^{2},
$$

and of the inequality [1]

$$
\begin{aligned}
& \int_{0}^{t} \int_{-\infty}^{\infty} \frac{\exp \left\{-\lambda|x-z|^{2} /(t-\tau)\right\}}{(t-\tau)^{\mu}} \frac{\exp \left\{-\lambda|z-y|^{2} / \tau\right\}}{\tau^{\nu}} d z d \tau \\
& \quad \leqq \text { const. } \frac{\exp \left\{-\lambda|x-y|^{2} / t\right\}}{t^{\mu+\nu-1-N / 2}}
\end{aligned}
$$

where $d z=d z^{1} \cdots d z^{N}$ and $\lambda>0, \mu<N / 2+1, \nu<N / 2+1$. The following, easily verified, inequality will also be used:

$$
\begin{aligned}
& \int_{-\infty}^{\infty} \exp \left\{-\lambda|x-z|^{2} / t\right\} \exp \left\{-\lambda|z-y|^{2} / t\right\} d z \\
& \quad \leqq \text { const. } \exp \left\{-\mu|x-y|^{2} / t\right\} t^{N / 2}
\end{aligned}
$$

where $d z=d z^{1} \cdots d z^{N}$ and $\lambda>\mu>0$. We shall denote by $A_{,}$constants which (unless otherwise stated) may depend only on $h$ and on the manifold $M$.

Using (3.6) one can prove by induction on $i$ that

$$
\left|\partial_{P}^{h} \delta_{m}^{i}(P, U, t)\right| \leqq \frac{A_{1}^{i+1}}{i !} t^{i(m+1-|h| / 2)-1-N / 2} e^{-r^{2} / 5 t}
$$


The case $i=1$ follows by (2.11), (2.12). (In deriving (3.8) we also use the elementality inequality $\lambda e^{-\alpha \lambda} \leqq$ const. $e^{-\delta \lambda}$ for all $\lambda>0$, where $\alpha, \delta$ are constants and $\alpha>\delta \geqq 0$.) In (3.8) it is understood that $t^{\circ}$ (if it occurs) must be replaced by $-\log t$. From now on we take $m$ such that

$$
m+1-\frac{|h|}{2}>0 \text {. }
$$

Using the definition (2.14) we then conclude from (3.8) that

$$
\left|\partial_{P}^{h} \gamma_{m}(P, Q, t)\right| \leqq A_{2} e^{-r^{2} / 5 t} t^{m-\left(\mid h_{1}+N\right) / 2} .
$$

Next, from the definition of $\Theta_{m}$ one derives

$$
\left|\partial_{P}^{h} \Theta_{m}(P, Q, t)\right| \leqq A_{3} e^{-r^{2} / 5 t} t^{-(|b|+N) / 2} .
$$

Combining (3.9) and (3.10) $(h=0)$ and applying (3.6), we get

$$
\left|\int_{0}^{t / 2}\left[\partial_{P}^{h} \gamma_{m}(P, W, \tau), \Theta_{m}\left(U, W, \frac{t}{2}-\tau\right)\right] d \tau\right| \leqq A_{4} e^{-2 r^{2} / 5 t} t^{m+1-(|m|+N) / 2}
$$

Using (3.10), (3.11) one easily derives, applying (3.7),

$$
J_{2}(P, t) \leqq A_{5} t^{m+1-|h|-N / 2} .
$$

Similary one gets

$$
J_{3}(P, t) \leqq A_{6} t^{2(m+1)-\mid h_{1-N / 2}} .
$$

Evaluation of $J_{1}(P, t)$. From the construction of $\Theta_{m}$ it follows that for every sufficiently small neighborhood $V$ we may take it to be of the form

$$
\Theta_{m}(P, U, t)=H_{m}(P, U, t)+R_{m}(P, U, t) \text { for all } P \in V
$$

where $H_{m}$ is constructed in $\S 2$ and where, for some $\alpha^{\prime}>0$,

$$
\left|\partial_{P}^{h} R_{m}(P, U, t)\right| \leqq A_{7} e^{-\alpha^{\prime} / t} t^{|h|+N / 2} \leqq A_{8} t^{\zeta}
$$

for any $\zeta>0 . A_{8}$ depends also on $\zeta$. Next,

$$
\partial_{P}^{h} H_{m}(P, U, t)=\sum_{j=0}^{m} t^{j} \sum_{|\nu|=0}^{|h|}\left(\begin{array}{l}
h \\
\nu
\end{array}\right) \partial_{P}^{\nu} f \partial_{P}^{h-\nu}\left(M U_{\jmath}\right)
$$

where $\left(\begin{array}{l}h \\ \nu\end{array}\right)=\left(\begin{array}{l}h_{1} \\ \nu_{1}\end{array}\right) \cdots\left(\begin{array}{l}h_{N} \\ \nu_{N}\end{array}\right)$. It is easily seen that

$$
\partial_{P}^{\nu} f\left(r^{2}, t\right)=\sum_{|\mu|=0}^{\nu_{0}} H_{\nu \mu}\left(\frac{y-x}{\sqrt{t}}\right) f\left(r^{2}, t\right) t^{|\nu| / 2+|\mu| / 2}
$$

where $y^{i}, x^{i}$ are the coordinates of $U, P$ respectively, and $H_{\nu \mu}(z)$ is a polynomial in $z=\left(z^{1}, \cdots, z^{N}\right)$ with $C^{\infty}$ coefficients which, for $H_{v 0}$, are 
functions of $x$ only. Substituting (3.17) into (3.16) and recalling that $M(P, U) \nu_{2}$ becomes $\left(\delta_{I}^{J}\right)$ at $P=U$, we obtain

$$
\partial_{P}^{h} H_{m}(P, U, t)=H_{n o}\left(\frac{y-x}{\sqrt{t}}\right) f\left(r^{2}, t\right) t^{-\mid h_{1} / 2} Y+S_{h}(P, U, t)
$$

where $Y$ is the matrix $\left(\delta_{I}^{J}\right)$ and

$$
\left|S_{h}(P, U, t)\right| \leqq A_{9} e^{-r^{2} / 2 t} t^{(1-|h|-N) / 2} .
$$

Combining (3.14), (3.15), (3.18), (3.19) we conclude that

$$
\partial_{P}^{h} \Theta_{m}(P, U, t)=H_{h 0}\left(\frac{y-x}{\sqrt{t}}\right) f\left(r^{2}, t\right) t^{-|h| / 2} Y+T_{h}(P, U, t)
$$

and

$$
\left|T_{h}(P, U, t)\right| \leqq A_{10} t^{\left(1-\mid h^{\prime}-N\right) / 2} .
$$

Using the definition of $J_{1}$, and substituting (3.20) in the part of the integral $\left[\partial_{P}^{h} \Theta_{m}(P, U, t / 2), \partial_{P}^{h} \Theta_{m}(P, U, t / 2)\right]_{U}$ taken over a coordinate patch $V_{0}$ containing $\bar{V}: y^{i}-x^{i}=\xi^{i} \sqrt{t}$, we find that

$$
J_{1}(P, t)=\left(C_{h}(P)+B_{0}(P, t)\right) t^{-|h|-N / 2}
$$

where $C_{h}(P)$ is a continuous function of $P$, and $\left|B_{0}(P, t)\right| \leqq A_{11} \sqrt{t}$ for $P \in V, 0<t \leqq b$, for any $b>0$. $A_{11}$ depends on $b$.

Combining the evaluation of $J_{1}$ with (3.12), (3.13), we obtain from (3.4),

$$
\sum_{i=1}^{\infty}\left|\partial^{h} \omega_{i}(P)\right|^{2} e^{-\lambda_{i} t}=C_{h}(P) t^{-|h|-N / 2}+D_{h}(P, t) t^{-|h|-(N-1) / 2}
$$

where $D_{h}(P, t)$ is a uniformly continuous function of $(P, t), P \in V$ and $0<t \leqq b$ for any $b>0$. Thus

$$
\left|D_{h}(P, t)\right| \leqq A_{12}
$$

where $A_{12}$ depends on $b$.

Note that the $A_{i}$, in particular $A_{12}$, are independent of $P$ which varies in $V$.

4. Asymptotic formulas. To derive asymptotic formulas from the equation (3.22) we use a Tauberian theorem due to Karamata, specialized to Dirichlet series [14; p. 192]. It states:

Let $a_{k} \geqq 0$ and $0 \leqq \lambda_{1} \leqq \lambda_{2} \leqq \cdots \leqq \lambda_{n} \leqq \cdots$, and assume that the Dirichlet series $f(t)=\sum_{k=1}^{\infty} a_{k} e^{-\lambda_{k} t}$ converges for $t>0$ and satisfies 


$$
f(t) \sim \frac{A}{t^{\gamma}} \text { as } t \searrow 0 \quad(\gamma \geqq 0) .
$$

Then the function $\alpha(x)=\sum_{\lambda_{k} \leqq x} a_{k}$ satisfies

$$
\alpha(x) \sim \frac{A x^{\gamma}}{\Gamma(\gamma+1)} \text { as } x \rightarrow \infty .
$$

Applying it to (3.22) (using (3.23)), we get

$$
\sum_{\lambda_{i} \leq \lambda}\left|\partial^{h} \omega_{i}(P)\right|^{2}=\frac{C_{h}(P)}{\Gamma(|h|+1+N / 2)} \lambda^{\mid h_{1+N / 2}[1+o(1)](\lambda \rightarrow \infty)}
$$

and $o(1) \rightarrow 0$ as $\lambda \rightarrow \infty$, uniformly in $P \in V$.

Let $\lambda_{1}=\cdots=\lambda_{q-1}=0, \lambda_{q}>0$. Using the asymptotic formula (4.1) we shall prove:

THEOREM 1. For any $h$ and for any $\varepsilon>0$, the series

$$
\sum_{i=q}^{\infty} \frac{\left|\partial^{h} \omega_{i}(P)\right|^{2}}{\lambda_{i}^{N / 2+|h|+\varepsilon}}
$$

is uniformly convergent in $P \in M$.

Proof. We introduce the function

$$
B(P, \lambda) \equiv \sum_{\lambda_{q} \leqq \lambda_{i} \leqq \lambda}\left|\partial^{h} \omega_{i}(P)\right|^{2} .
$$

Then, we can write the series (4.2) in the form

$$
\int_{\lambda^{\prime}}^{\infty} \frac{d B(P, \lambda)}{\lambda^{N / 2+|h|+\varepsilon}} \text { for any } 0<\lambda^{\prime}<\lambda_{q} .
$$

Integrating by parts we get

$$
\lim _{\mu \rightarrow \infty}\left[\frac{B(P, \lambda)}{\lambda^{N / 2+|h|+\varepsilon}}\right]_{\lambda=\lambda \prime}^{\lambda=\mu}-\left(\frac{N}{2}+|h|+\varepsilon\right) \int_{\lambda,}^{\infty} \frac{B(P, \lambda)}{\lambda^{N / 2+|h|+\varepsilon+1}} d \lambda .
$$

Since, by (4.1), $B(P, \lambda) \leqq A_{13} \lambda^{|n|+N / 2}$ and since $B\left(P, \lambda^{\prime}\right)=0$, the first term in (4.3) vanishes. The integral in (4.3) converges uniformly in $P$ in view of the bound on $B(P, \lambda)$ just given. The proof of Theorem 1 is thereby completed.

5. Solution of the system (1.1), (1.2). We first derive the formal solution. Substituting

$$
g(P)=\sum_{n=1}^{\infty} g_{n} \omega_{n}(P), h(P)=\sum_{n=1}^{\infty} h_{n} \omega_{n}(P), f(P, t)=\sum_{n=1}^{\infty} f_{n}(t) \omega_{n}(P)
$$




$$
v(P, t)=\sum_{n=1}^{\infty} v_{n}(t) \omega_{n}(P)
$$

into (1.1), (1.2) we arrive at the equations

$$
\begin{aligned}
& v_{n}^{\prime \prime}(t)+\lambda_{n} v_{n}(t)=f_{n}(t) \\
& v_{n}(0)=g_{n}, v_{n}^{\prime}(0)=h_{n} .
\end{aligned}
$$

If $\lambda_{n}=0$ the solution is

$$
v_{n}(t)=g_{n}+h_{n} t+\int_{0}^{t} f(\tau)(t-\tau) d \tau .
$$

If $\lambda_{n}>0$ the solution is

$$
v_{n}(t)=g_{n} \cos \sqrt{\lambda_{n}} t+\frac{h_{n}}{\sqrt{\lambda_{n}}} \sin \sqrt{\lambda_{n}} t+\frac{1}{\sqrt{\lambda_{n}}} \int_{0}^{t} f_{n}(\tau) \sin \sqrt{\lambda_{n}}(t-\tau) d \tau .
$$

Hence, the formal solution of (1.1), (1.2) is

$$
\begin{aligned}
& v(P, t)=\sum_{n=1}^{\infty} g_{n} \omega_{n}(P) \cos \sqrt{\lambda_{n}} t+\sum_{n=1}^{q-1} h_{n} \omega_{n}(P) t \\
& \quad+\sum_{n=q}^{\infty} \frac{h_{n}}{\sqrt{\overline{\lambda_{n}}}} \omega_{n}(P) \sin \sqrt{\lambda_{n}} t+\sum_{n=1}^{q-1} \omega_{n}(P) \int_{0}^{t} f_{n}(\tau)(t-\tau) d \tau \\
& \quad+\sum_{n=q}^{\infty} \frac{1}{\sqrt{\bar{\lambda}_{n}}} \omega_{n}(P) \int_{0}^{t} f_{n}(\tau) \sin \sqrt{\lambda_{n}}(t-\tau) d \tau .
\end{aligned}
$$

To prove that the formal solution is a genuine one we observe that if $\lambda_{n}>0$

$$
g_{n}=\int_{M} g(Q) * \omega_{n}(Q)=\frac{1}{\lambda_{n}^{m}} \int_{M} L^{m} g(Q) * \omega_{n}(Q)
$$

for any positive integer $m$. Applying Bessel's inequality, we get

$$
\sum_{n=1}^{\infty} \lambda_{n}^{2 m} g_{n}^{2} \leqq \int_{M} L^{m} g(Q) * L^{m} g(Q)=\left\|L^{m} g\right\|^{2} .
$$

Similarly,

$$
\sum_{n=1}^{\infty} \lambda_{n}^{2 m} h_{n}^{2} \leqq\left\|L^{m} h\right\|^{2}, \sum_{n=1}^{\infty} \lambda_{n}^{2 m}\left(f_{n}(t)\right)^{2} \leqq\left\|L^{m} f(\cdot, t)\right\|^{2} .
$$

It will be enough to show that the part of the first series on the right side of (5.5), where summation is on $\lambda_{n}>0$, when differentiated term-by-term twice with respect to $P$ is uniformly convergent in $P \in M$, $0 \leqq t \leqq b$, for any $b>0$. Now the series obtained is majorized by

$$
\Sigma\left|g_{n}\right|\left|\partial^{2} w_{n}(P)\right| \leqq \Sigma \lambda_{n}^{k}\left|g_{n}\right| \frac{\left|\partial^{2} \omega_{n}(P)\right|}{\lambda_{n}^{k}} \leqq \Sigma \lambda_{n}^{2 k} g_{n}^{2} \Sigma \frac{\left|\partial^{2} \omega_{n}(P)\right|^{2}}{\lambda_{n}^{2 k}}
$$


Hence that series is uniformly convergent if $k>N / 2+1$.

It is clear that each series in (5.5) can actually be differentiated term-by-term any number of times and the resulting series is uniformly convergent.

By a solution of (1.1), (1.2) we mean a $p$-form which is $(a)$ twice continuously differentiable in $(P, t)$ for $P \in M, t>0(b)$ once continuously differentiable in $t$ for $P \in M, t \geqq 0$ and (c) satisfies (1.1), (1.2).

The uniqueness of the solution can be proved as for the classical wave equation. Assuming $g \equiv 0, h \equiv 0, f \equiv 0$ and using the rule $\int d u^{*} \omega=\int u^{*} \delta \omega$ one finds that if $u$ is a solution then

$$
\frac{\partial}{\partial t} \int_{M}\left[u_{t} * u_{t}+\delta u * \delta u+d u * d u-c u * u\right]=0 .
$$

Since the integral vanishes for $t=0$, it vanishes for all $t>0$. Since the integrand is nonnegative, $u_{t} * u_{t} \equiv 0$, which implies $u_{t} \equiv 0$ and hence, $u \equiv 0$.

We have thus completed the proof of the following theorem.

THEOREM 2. Let $g, h$ be $C^{\infty} p$-forms and let $f$ be a $C^{\infty} p$-form such that $\partial_{P}^{\lambda} f$ is continuous in $(P, t)$, for any $\lambda$. Then the Cauchy problem (1.1), (1.2) has one and only one solution. The solution is a $C^{\infty} p$-form and is given by (5.5).

The assumption that the manifold $M$ is $C^{\infty}$ can be weakened. Indeed, the theory of differential forms used above remains valid under the assumption that the metric tensor is $C^{5}$ (Gaffney [3]; see also Friedrichs [2]). The assumptions on $f, g, h$ can also be weakened without any modification of the preceding proof of Theorem 2 .

We need the assumptions:

(A) The metric tensor $g_{i j}$ belongs to $C^{[N / 2]+2}$ and to $C^{5}$, and $c$ belongs to $C^{[N / 2]+1}$ (recall that $c \leqq 0$ ).

(B) The form $g$ belongs to $C^{[N / 2]+3}$ and $L^{[(N+4) / 4]} g$ belongs to $C^{1}$.

(C) The form $h$ belongs to $C^{[N / 2]+2}$ and $L^{[(N+2) / 2]} h$ belongs to $C^{1}$.

(D) The form $f$ and its first $[N / 2]+2 p$-derivatives are continuous for $P \in M, 0 \leqq t \leqq b$ (for any $b>0$ ); $L^{[(N+2) / 2]} f$ and its first $p$-derivatives are continuous for $P \in M, 0 \leqq t \leqq b$.

THEOREM 2'. Under the assumptions (A) - (D), there exists one and only one solution of the Cauchy problem (1.1), (1.2). It is given by (5.5).

The assertion of Theorem 2' remains valid if we further weaken the assumptions (A) - (D) by replacing the classes of continuous deriva- 
tives $C^{q}$ by classes of "strong" derivatives $W_{2}^{q}$ (see [6]), assuming that $g_{i j} \in C^{5}$.

6. The heat equation. The method of $\S 5$ can easily be extended to solve the system (1.4), (1.5). The formal solution is

$$
u(P, t)=\sum_{n=1}^{\infty} g_{n} \omega_{n}(P) e^{-\lambda n^{t}}+\sum_{n=1}^{\infty} \omega_{n}(P) \int_{0}^{t} f_{n}(\tau) e^{-\lambda_{n}(t-\tau)} d \tau .
$$

We shall need the assumptions:

(A') $g_{i}$, belong to $C^{[N / 2]+1}$ and to $C^{5}$, and $e$ belongs to $C^{[N / 2]}$.

(B') The form $g$ belongs to $C^{[N / 2]+1}$ and $L^{[N / 4]} g$ belongs to $C^{1}$.

THEOREM 3. Under the assumption ( $\left.\mathrm{A}^{\prime}\right),\left(\mathrm{B}^{\prime}\right)$, (D) there exists a unique solution of the system (1.4), (1.5). It is given by (6.1).

REMARK 1. The assumption $c \leqq 0$ is not needed for the validity of Theorem 3 since it can be achieved by a transformation $u=e^{\alpha t} u$ for any constant $\alpha \geqq c$.

REMARK 2. Assuming $c \leqq 0, f \equiv 0$, we can rewrite (6.1) as an operator equation

$$
T_{t}=H+\sum_{k=1}^{\infty} e^{-\mu_{k} t} H_{k}
$$

where $\left\{\mu_{k}\right\}$ is the sequence $\left\{\lambda_{j}\right\}$ taken without multiplicities, $H_{k}$ is the projection into the space of eigenforms corresponding to $\mu_{k}, H$ corresponds to $\mu_{0}=0$, and $T_{t}$ is the operator which maps $g$ into the solution $u$, that is, $u(P, t)=T_{t} g(P)$. Formula (6.2) was derived, in a different way (for $c \equiv 0$ ) by Milgram and Rosenbloom [10].

\section{REFERENCES}

1. F. G. Dressel, The fundamental solution of the parabolic equation II, Duke Math. J., 13 (1946), 61-70.

2. K. O. Friedichs, Differential forms on Riemannian manifolds, Comm. Pure Appl. Math., 8 (1955), 551-590.

3. M. P. Gaffney, Hilbert space methods in the theory of harmonic integrals, Trans. Amer. Math. Soc., 78 (1955), 426-444.

4. M. P. Gaffney, Asymptotic distributions associated with the Laplacian forms, Comm. Pure Appl. Math., 11 (1958), 535-545.

5. L. Garding, Cauchy's Problem for hyperbolic equations, University of Chicago Notes, 1957.

6. V. A. Il'in, On solving mixed problems for hyperbolic and parabolic equations, Uspehi Math. Nauk, 15, no. 2 (1960), 97-154.

7. K. Kodaira, Harmonic fields in Riemannian manifolds, generalized potential theory, Ann. of Math., 50 (1949), 587-665.

8. O. A. Ladyzhenskaya, The mixed problem for hyperbolic equations, Gosudarvstv. Izdat. 
Tehn.-Teor. Lit., Moscow, 1953.

9. J. Leray, Lectures on hyperbolic equations with variable coefficients, Princeton, Inst. for Advanced Study, 1952.

10. A. N. Milgram and P. C. Rosenbloom, Harmonic forms and heat conduction I: Closed Riemannian manifolds, Proc. Nat. Acad. Sci., 37 (1951), 180-184.

11. S. Minakshisundaram, Eigenfunctions on Riemannian manifolds, J. Indian Math. Soc., 17 (1953), 159-165.

12. I. G. Petrowski, Über des Cauchysche Problem für Systeme von partiellen Differentialgleichungen, Rec. Math. (Moscow) N.S., 2 (1937), 814-868.

13. G. De Rham, Variétés Différentiables, Hermann, Paris, 1955.

14. D. Widder, The Laplace Transform, Princeton Univ. Press, Princeton, 1946.

UNIVERSITY OF MINNESOTA

INSTITUTE OF TECHNOLOGY

MINNEAPOLIS, MINNESOTA 



\section{PACIFIC JOURNAL OF MATHEMATICS}

\section{EDITORS}

\author{
RaLPh S. Phillips \\ Stanford University \\ Stanford, California \\ F. H. BRowNELL \\ University of Washington \\ Seattle 5 , Washington
}

A. L. Whiteman

University of Southern California

Los Angeles 7, California

L. J. Paige

University of California

Los Angeles 24, California

\author{
E. F. BECKENBACH \\ T. M. CHERRY
}

\author{
ASSOCIATE EDITORS

$\begin{array}{lll}\text { D. DERRY } & \text { H. L. ROYDEN } & \text { E. G. STRAUS } \\ \text { M. OHTSUKA } & \text { E. SPANIER } & \text { F. WOLF }\end{array}$

\section{SUPPORTING INSTITUTIONS}

\author{
UNIVERSITY OF BRITISH COLUMBIA \\ CALIFORNIA INSTITUTE OF TECHNOLOGY \\ UNIVERSITY OF CALIFORNIA \\ MONTANA STATE UNIVERSITY \\ UNIVERSITY OF NEVADA \\ NEW MEXICO STATE UNIVERSITY \\ OREGON STATE COLLEGE \\ UNIVERSITY OF OREGON \\ OSAKA UNIVERSITY \\ UNIVERSITY OF SOUTHERN CALIFORNIA
}

\author{
STANFORD UNIVERSITY \\ UNIVERSITY OF TOKYO \\ UNIVERSITY OF UTAH \\ WASHINGTON STATE COLLEGE \\ UNIVERSITY OF WASHINGTON \\ AMERICAN MATHEMATICAL SOCIETY \\ CALIFORNIA RESEARCH CORPORATION \\ HUGHES AIRCRAFT COMPANY \\ SPACE TECHNOLOGY LABORATORIES \\ NAVAL ORDNANCE TEST STATION
}

Mathematical papers intended for publication in the Pacific Journal of Mathematics should be typewritten (double spaced), and the author should keep a complete copy. Manuscripts may be sent to any one of the four editors. All other communications to the editors should be addressed to the managing editor, L. J. Paige at the University of California, Los Angeles 24, California.

50 reprints per author of each article are furnished free of charge; additional copies may be obtained at cost in multiples of 50 .

The Pacific Journal of Mathematics is published quarterly, in March, June, September, and December. The price per volume (4 numbers) is $\$ 12.00$; single issues, $\$ 3.50$. Back numbers are available. Special price to individual faculty members of supporting institutions and to individual members of the American Mathematical Society: $\$ 4.00$ per volume; single issues, $\$ 1.25$.

Subscriptions, orders for back numbers, and changes of address should be sent to Pacific Journal of Mathematics, 103 Highland Boulevard, Berkeley 8, California.

Printed at Kokusai Bunken Insatsusha (International Academic Printing Co., Ltd.), No. 6, 2-chome, Fujimi-cho, Chiyoda-ku, Tokyo, Japan.

\section{PUBLISHED BY PACIFIC JOURNAL OF MATHEMATICS, A NON-PROFIT CORPORATION}

The Supporting Institutions listed above contribute to the cost of publication of this Journal, but they are not owners or publishers and have no responsibility for its content or policies.

Reprinted 1966 in the United States of America 


\section{Pacific Journal of Mathematics}

\section{Vol. 11, No. 4}

A. V. Balakrishnan, Prediction theory for Markoff processes . . . . . . . . . . 1171

Dallas O. Banks, Upper bounds for the eigenvalues of some vibrating systems . . . . 1183

A. Białynicki-Birula, On the field of rational functions of algebraic groups ...... 1205

Thomas Andrew Brown, Simple paths on convex polyhedra .............. 1211

L. Carlitz, Some congruences for the Bell polynomials . . . . . . . . . . . . 1215

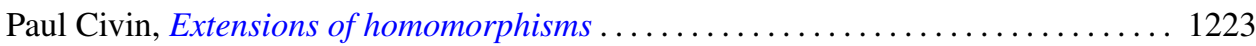

Paul Joseph Cohen and Milton Lees, Asymptotic decay of solutions of differential

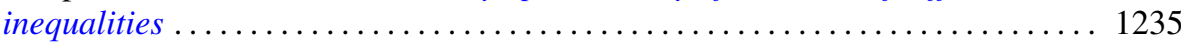

István Fáry, Self-intersection of a sphere on a complex quadric . . . . . . . . . . 1251

Walter Feit and John Griggs Thompson, Groups which have a faithful representation

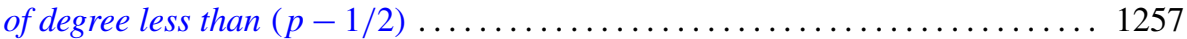

William James Firey, Mean cross-section measures of harmonic means of convex

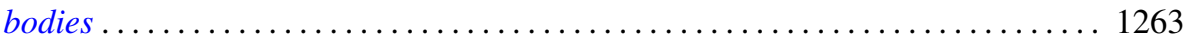

Avner Friedman, The wave equation for differential forms . . . . . . . . . . 1267

Bernard Russel Gelbaum and Jesus Gil De Lamadrid, Bases of tensor products of

Banach spaces ................................... 1281

Ronald Kay Getoor, Infinitely divisible probabilities on the hyperbolic plane . . . . 1287

Basil Gordon, Sequences in groups with distinct partial products . . . . . . . . . . . . 1309

Magnus R. Hestenes, Relative self-adjoint operators in Hilbert space . . . . . . . . . 1315

Fu Cheng Hsiang, On a theorem of Fejér ......................... 1359

John McCormick Irwin and Elbert A. Walker, On N-high subgroups of Abelian

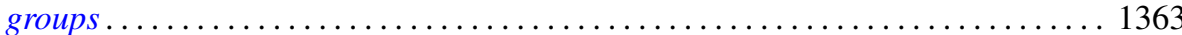

John McCormick Irwin, High subgroups of Abelian torsion groups . . . . . . . . . 1375

R. E. Johnson, Quotient rings of rings with zero singular ideal . . . . . . . . . . . 1385

David G. Kendall and John Leonard Mott, The asymptotic distribution of the time-to-escape for comets strongly bound to the solar system ...

Kurt Kreith, The spectrum of singular self-adjoint elliptic operators ....

Lionello Lombardi, The semicontinuity of the most general integral of the calculus of variations in non-parametric form ................................

Albert W. Marshall and Ingram Olkin, Game theoretic proof that Chebyshev inequalities are sharp

Wallace Smith Martindale, III, Primitive algebras with involution . . William H. Mills, Decomposition of holomorphs ..............

James Donald Monk, On the representation theory for cylindric algebras . . . . . . 1447

Shu-Teh Chen Moy, A note on generalizations of Shannon-McMillan theorem . . . . 1459

Donald Earl Myers, An imbedding space for Schwartz distributions . .

John R. Myhill, Category methods in recursion theory .........

Paul Adrian Nickel, On extremal properties for annular radial and circular slit mappings of bordered Riemann surfaces

Edward Scott O'Keefe, Primal clusters of two-element algebras . .

Nelson Onuchic, Applications of the topological method of Wazewski to certain

problems of asymptotic behavior in ordinary differential equations ...

Peter Perkins, A theorem on regular matrices................

Clinton M. Petty, Centroid surfaces .... 